\title{
LUCES Y SOMBRAS DE LA ECONOMÍA FEMINISTA. REPASO DESDE SUS BASES HASTA LA ACTUALIDAD
}

\author{
LIGHTS AND SHADOWS OF FEMINIST ECONOMICS.
}

REVIEW FROM ITS BASES TO THE PRESENT

Lorena García Saiz

Doctoranda. Universitat Jaume I

\section{RESUMEN}

El movimiento feminista apuesta por una «economía feminista», donde la vida y los cuidados sean el puntal de la estructuración y el desarrollo social. Un claro ejemplo de movilización desde esa óptica viene siendo la realización de una huelga feminista en España el 8 de marzo de cuidados, de consumo, laboral, estudiantil y asociativa, un desafío para seguir tratando de hacer brechas en un sistema capitalista que agoniza pero sigue mutando para sobrevivir. El repaso por las aportaciones de diversas corrientes feministas como base de esta «economía feminista» hasta la actualidad, ayuda a entender tanto las dificultades encontradas como los logros obtenidos en una lucha más activa que nunca y marcada por un claro objetivo: la consecución de un mundo más justo e igualitario.

Palabras Clave: economía feminista, género, cuidados, capitalismo, patriarcado.

\section{ABSTRACT}

The feminist movement is committed to a "feminist economy», where life and care are the mainstay of structuring and social development. A clear example of mobilization from this perspective is the realization of a care, consumption, labor, student and associative feminist strike in Spain on 8 March, a challenge to continue trying to make gaps in a capitalist system that is dying but it keeps mutating to survive. The review of the contributions of various feminist currents as the basis of this «feminist economy» to the present time, helps to understand both the difficulties encountered and the achievements obtained in a fight more active than ever and marked by a clear objective: the achieving a more just and egalitarian world.

Keywords: feminist economics, gender, care, capitalism, patriarchy. 


\section{Introducción}

El pasado 8 de marzo de 2019 fueron miles las mujeres y también hombres quienes salieron a la calle en España con motivo de la convocatoria de una huelga de cuidados, consumo, estudiantil, laboral y asociativa bajo el lema Si nosotras paramos, se para el mundo. De esta manera, se buscaba denunciar como las mujeres son pieza clave para el sostenimiento de un capital y un patriarcado que descentra la verdadera razón de ser de las personas al ser invisibilizadas: los cuidados y la vida. Sin los cuidados no hay vida y esta idea debería ser el eje para garantizar un mundo más justo e igualitario para todos los seres humanos, sin ningún tipo de opresión ni sometimiento, mediante alternativas al actual sistema económico neoliberal. Estas reflexiones se han ido modelando desde hace unas décadas a través de la labor del movimiento feminista que, desde su segunda ola, experimenta un interés por analizar los motivos de la opresión de la mujer, haciendo especial hincapié en el análisis económico.

Es a partir de la década de los 70 cuando despierta un especial interés la producción doméstica, por lo que comienza a desarrollarse un corpus de análisis y creación de lo que hoy se conoce como «economía feminista», para hacer frente a un capitalismo que ha ido mutando para sobrevivir, pero del que podemos decir que «la heterosexualidad es su estructura social, el deseo su dinámica interna, el género y la familia sus formas quietas, los roles sexuales sus cualidades generalizadas en la persona social, la reproducción una consecuencia y el control su lucha» (Mackinnon, 1989: 24). Ante la lectura de la economía neoclásica, que se sostiene bajo la división sexual del trabajo, diversas corrientes del Feminismo empiezan a analizar la cuestión de la mujer y los elementos que la oprimen.

\section{Aportaciones de los Feminismos a la "economía feminista»}

Desde el feminismo radical, consideran que la «raíz» de la opresión de las mujeres está en el seno del sistema patriarcal, donde los hombres obtienen ventajas de la subordinación femenina y subraya que el resto de formas de explotación, jerarquía, supremacía, no son más que extensiones de la supremacía masculina. Así, el patriarcado antes que el capitalismo, el racismo o el colonialismo, representa el enemigo común y principal de todas las mujeres. Aparecen varios textos significativos como La dialéctica del Sexo (1970) de Shulamith Firestone, en el que identifica la diferencia biológica entre hombres y mujeres como la raíz de la subordinación femenina. Señala que los objetivos del programa radical consisten en una revuelta de las mujeres para controlar los medios de reproducción que será paralela 
a la revolución del proletariado para la apropiación colectiva de los medios de producción. Cuando esto ocurra se llegará no ya a la igualdad, ni la abolición de privilegios culturales, sino a algo más radical que llama «neutralización cultural de las diferencias genitales entre los humanos» (Amorós, 2005: 84-85), por lo que las diferencias sexuales no tendrán ninguna traducción cultural, siendo el final de todo sistema sexo/género. Esto dio origen a un feminismo socialista liberado de las tesis marxistas sobre la «condición femenina» (Puleo, 2005: 64), una idea que queda recogida en la propuesta de Sistema Dual que Heidi Hartmann elabora -basado en la adaptación del patriarcado a los diversos sistemas de organización social, particularmente al capitalismo, en el que se producen pactos interclasistas para mantener la situación de subordinación de las mujeres-y que se verá más adelante.

También destaca Sisterhood is Powerful (1970) de Morgan, donde habla del concepto de sororidad como elemento común de todas las mujeres ante la opresión del sexismo, que para Mitchell era la raíz del resto de opresiones -capitalismo, racismo e imperialismo-, tal y como lo recoge en Womens's State (1971). Ahonda en la necesidad de transformar las cuatro estructuras en las que se integran las mujeres -producción reproducción, sexualidad y socialización- para favorecer su liberación.

Por su parte, Millet publica Política sexual (1970), para combatir los prejuicios patriarcales arraigados, incluso entre la izquierda, e impulsar líneas de actuación más radicales y renovadoras. El patriarcado se define como «política sexual», entendido como «conjunto de estratagemas destinadas a mantener un sistema» (Millet, 1995: 67). Por tanto, la relación entre los sexos es política, es una relación de poder. Considera que uno de los instrumentos más eficaces del gobierno patriarcal es el control económico sobre las mujeres, que han realizado siempre tareas más rutinarias o pesadas, pero el problema se centra en su retribución económica, un debate que las feministas marxistas Dalla Costa y James sacan a la luz con su publicación El poder de las mujeres y la subversión de la comunidad (1972).

Para las feministas marxistas, el capitalismo es el que ha transformado el papel y la estructura de la familia, creando una familia nuclear heterosexual que niega su papel como unidad productiva y relega a la mujer a una posición subordinada y un rol de reproducción de la fuerza de trabajo, al ser el hombre el que cuenta con un salario suficiente para mantener a la mujer e hijos. De este modo, se establece un contrato de trabajo estipulado entre el capitalista y el «padre de familia que es a la vez contrato laboral y sexual, ya que otorga a los hombres el libre acceso al cuerpo de sus mujeres e hijos» (Arruzza, 2015: 114). Por ello, consideran que es necesario que se retribuya el trabajo doméstico con un salario, para que «el trabajo reproductivo de la fuerza de trabajo fuera reconocido abiertamente como trabajo productivo y dejara de ser retribuido por vía indirecta, a través del salario de su marido» (Arruzza, 2015: 114). 
Desde el feminismo materialista, Delphy establece en El enemigo principal (1982) las bases para un análisis de la explotación de la mujer a la que llama «explotación patriarcal», como consecuencia del control de la fuerza reproductora de la mujer mediante la explotación sexual. Para Delphy no es el capitalismo quien se apropia del trabajo doméstico sino los hombres, que son los beneficiarios directos del trabajo productivo/reproductivo de la mujer (marido, padre, hermano). Considera necesario analizar ambas explotaciones porque en ellas actúa la relación entre el capitalismo y el patriarcado. Afirma que, junto al modo de producción capitalista, existe el de producción patriarcal, que determina las relaciones de producción entre hombres y mujeres y se basa en la apropiación integral por parte de los hombres del trabajo doméstico de las mujeres. Así, en esta relación de producción, hombres y mujeres son clases antagonistas y ligadas por una relación de explotación. Aparece por primera vez la consideración de las mujeres como clase social -no sexual como señala Firestone- y el análisis del trabajo doméstico como trabajo productivo (Oliva, 2005), cuya prestación de estos servicios gratuita se quiere mantener desde el entramado patriarcal. Reconoce que el modo doméstico de producción es la base económica del patriarcado y el más difundido a escala mundial.

Por tanto, los análisis llevados a cabo por Delphy y Dalla Costa, contradicen al marxismo ortodoxo, acusado de subestimar la función del trabajo reproductivo y de negar su papel reproductivo, lo reinterpretan y pasan a considerar el trabajo doméstico como productor de mercancías y valor, pasando a ser un trabajo productivo que no es retribuido.

Hartmann calificó la relación entre marxismo y feminismo en el artículo The Unhappy Marriage of Marxism and Feminism (1981) de «matrimonio desgraciado». De aquí surge gran parte del desarrollo de la teoría feminista socialista norteamericana, subrayando como la "cuestión de la mujer no ha sido nunca para el marxismo clásico una cuestión feminista», ya que se la define en sus relaciones de trabajo como clase desposeída (Molina, 2005: 161). Hartmann afirma que el patriarcado es anterior al capitalismo y que los hombres usaron el capitalismo para asegurar su situación de privilegio. Cree que el trabajo doméstico existe por los beneficios que obtiene el capital, tanto en la esfera de producción capital como en la de reproducción doméstica. La actividad de las mujeres está basada en las necesidades del capitalismo, ya que las mujeres son mano de obra flexible y, por otro lado, con el trabajo doméstico que realizan se reduce el valor de la fuerza de trabajo, lo que repercute en un mayor beneficio para el capital (Carrasco, 2017: 28).

Así, Hartmann o Delphy analizaban la opresión en una estructura de dos sistemas separados o Teoría Dual: capitalismo y patriarcado, modo de producción y reproducción sistema de clases y sistema de géneros (Carrasco, 2017: 26). Pero este análisis es insuficiente al 
ser difícil de integrar ambos sistemas en una estructura coherente no funcionalista que diera cuenta acerca de cómo se construyen las relaciones de género en la familia y en la producción

Frente a esta Teoría Dual, Young en su artículo Socialist Feminism and the Limits of Dual System Theory (1980), defiende una teoría unitaria: el Feminismo materialista histórico, en la que patriarcado y capitalismo son elementos del mismo sistema y las jerarquías de género, cambios en las relaciones de género e ideologías se consideran aspectos centrales de cualquier formación social. Se concibe como una «teoría de la totalidad de las relaciones sociales que toma las características de género como un atributo clave de esas relaciones» (Molina, 2005: 170), que dé cuenta de toda relación de opresión al incluir también la particular opresión de la mujer.

El feminismo marxista usa las principales herramientas de dicha teoría para hacer una lectura crítica. Busca comprender las relaciones bajo las que se desarrolla la actividad doméstica, su reconocimiento como «trabajo», las relaciones que mantiene con la producción capitalista y quienes se benefician de la existencia de este trabajo. «En este momento de crítica a Marx, nosotras usábamos a Marx, Marx nos dio herramientas para criticarlo» (Federici, 2017: 18).

En este sentido, Arruzza y Cirillo resaltan como la fuerza del feminismo marxista residen «en la centralidad que éste concede a la cuestión de las relaciones sociales capitalistas y al análisis del modo en que el capitalismo produce y sigue regenerando la opresión de género» (2017: 154) y resalta los tres puntos del debate desarrollados: trabajo doméstico y explotación capitalista; teoría unitaria y producción social; la relación histórica entre reificación de la identidad sexual y acumulación capitalista.

Respecto al primer punto -trabajo doméstico y explotación capitalista-, ya hemos visto como las marxistas feministas (Dalla Costa y James, 1970) denuncian como Marx solo tomó en cuenta en su análisis del valor de la fuerza de trabajo, el valor de las mercancías necesarias para su reproducción, pero no el valor doméstico desarrollado principalmente por las mujeres. Ante esto, ambas autoras consideran que el trabajo reproductivo desarrollado en las familias por las mujeres crea valor, ya que contribuye a constituir el valor de la mercancía que es la fuerza del trabajo.

Frente a esto, otras feministas marxistas señalan que el valor reproductivo contribuye a crear valor indirectamente al reproducir la fuerza de trabajo. Afirman que no produce valor directo, al desarrollarse dentro de un espacio como las relaciones interpersonales y el hogar, que no estaban incluidos directamente en el ciclo de producción y realización del valor.

Pese a la variedad de posturas dentro del feminismo marxista, es clave ver como las reflexiones que realiza sirven para mostrar que el trabajo de cuidados no remunerado desempeñado por las mujeres debe ser reconocido como trabajo, reivindicado políticamente. 
En cuanto al segundo punto, desde el movimiento marxista se apuesta tras varios procesos históricos por la teoría unitaria (Young, 1980). Se considera al capitalismo no como un sistema simplemente económico, sino como un conjunto que incluye un modo de producción y una forma de sociedad, como una relación social. Incide en analizar la perduración de la opresión de género en las sociedades del capitalismo avanzado. «Estos mecanismos son los de la división del trabajo, pero sobre todo los de la separación entre reproducción social y producción y de la subordinación de la reproducción social a la producción» (Arruzza y Cirillo, 2017: 157).

Dicha reproducción social es definida como un conjunto de actividades, comportamientos, emociones, relaciones e instituciones que están directamente implicadas en el mantenimiento de la vida cotidiana y es intergeneracional. Incluye la parte realizada mediante instituciones públicas o a través del mercado lque cuentan con una gran feminización en dicho sector), y también se ha ampliado para comprender la disponibilidad de medios elementales de supervivencia como el agua o el aire. Esto supone ver los efectos del capitalismo en la reproducción social y en el trabajo de reproducción realizado por las mujeres desde el punto de vista ecológico (Arruzza y Cirillo, 2017: 157-158). Es un proceso dinámico que implica la reproducción biológica y de la fuerza de trabajo, la reproducción de bienes de cambio y de producción, y la reproducción de las relaciones de producción (Carrasco, 2017: 35).

En cuanto a la tercera aportación del feminismo marxista, contribuye a analizar el modo en que las orientaciones y las preferencia sexuales se han reificado en identidades que corresponden con algunas dinámicas históricas dadas en los países del capitalismo avanzado. Es el caso de la difusión del consumo de masas, la descalificación del trabajo fabril y asalariado o la nueva centralización lograda por el tiempo libre y su organización bajo formas mediadas por mercancías en la definición de identidad persona, de género y sexual. Frente a esto, Arruzza y Cirillo hablan de apostar por recuperar «la centralidad del tema de la sexualidad para la articulación de una política que sea al mismo tiempo feminista, antirracista y de clase» (2017: 159).

La definición de trabajo productivo para Marx estaba relacionado con los modos de producción. Diferenció el trabajo productivo en sentido general -con el que se producía bienes y servicios socialmente útiles (valores de uso)- y el trabajo productivo en el contexto del modo de producción capitalista, con el que se producen mercancías con un valor de cambio -tanto si eran bienes como servicios-, que podían venderse con un beneficio. Por tanto, «que el trabajo fuese o no productivo dependía únicamente de si era intercambiado por el capital para producir un valor excedente y no de la naturaleza del trabajo mismo ni del producto» (Gardiner, 2017: 82). 
El marxismo ahondó más que la economía clásica en explorar las relaciones sociales que sirven de soporte para la actividad económica pero, al focalizar principalmente en la producción capitalista, no supo reconocer el papel del trabajo doméstico en la reproducción de la economía. Vio el hogar solo como lugar de consumo, y no como de producción de mercancías. «Esta insuficiencia fue la que generó el debate sobre el trabajo doméstico» (Gardiner, 2017: 86). He aquí la paradoja del marxismo: al abordar el conflicto con el capital en el ámbito mercantil, se oculta el lugar donde realmente se resuelve el conflicto en toda su esencia, no solo con el trabajo asalariado, sino con la vida misma. Al centrarse en la producción y, por ende, desatender y despolitizar el ámbito de la reproducción, se colabora con «la invisibilización que permite que la tensión no estalle, porque no se politiza. Esto muestra de forma tremendamente elocuente la limitada potencia de toda lucha anticapitalista que no se atreva a ahondar en la dimensión heteropatriarcal del capitalismo» (Pérez, 2014: 202-203).

Así, pese a que el feminismo marxista limitó su análisis principalmente a las mujeres en casa y estuvo marcado por un fuerte carácter economicista, no ahondó en el análisis e identificación en las relaciones de género presentes en la producción doméstica. Al igual que el sistema neoclásico oculta la posibilidad de conflicto en el seno familiar, en el sistema marxista el supuesto de la prioridad de clase oculta los conflictos posibles entre personas de la misma clase social (Carrasco, 2017: 44).

Esto no permite el análisis de las diferencias de género, ya que se sigue centrando principalmente en el «homus economicus» y no en aspectos como la responsabilidad o la dependencia, que son claves para comprender comportamientos y desarrollar «nuevos esquemas conceptuales que integren «supuestos tanto de individualidad como de interrelación influenciados ambos por el medio social» (Carrasco, 2017: 45).

Pero, por otro lado, este debate fue útil al colocar en la agenda una serie de aspectos hasta ese momento no tratados: el hogar como unidad de producción y no sólo como unidad de consumo; el estudio de la posición de las mujeres como amas de casa; el papel del trabajo doméstico en la reproducción del sistema social; el trabajo doméstico -y no sólo el salariocomo elemento determinante de los estándares de vida; la necesidad de ampliar las fronteras de la disciplina económica más allá de la producción de mercado; la identificación de relaciones sociales de producción en el hogar distintas a las que tienen lugar en la producción de mercado y lo inapropiado de intentar aplicar de forma mecánica instrumentos conceptuales elaborados para el análisis de la producción mercantil, a la producción doméstica. En cualquier caso, el legado más importante de la polémica fue la apertura de nuevas vías de análisis, lo que «permitió abordar aspectos que iban más allá de un análisis netamente económico del trabajo doméstico, donde las categorías marxistas se mostrarían insuficientes» (Carrasco, 2006: 12). 
Con la reformulación de las herramientas marxistas, el feminismo paso de interpretar el conflicto entre capital y trabajo asalariado a ser entre el capital contra todos los trabajos, tanto asalariado como el que se hace fuera de los circuitos de acumulación del capital. Como subraya Pérez

en un sentido más hondo, enfrenta al capital con la vida. Afirmamos que existe una contradicción estructural entre el proceso de valorización de capital y el proceso de sostenibilidad de la vida y que, bajo la preeminencia del primer proceso, el segundo está siempre bajo amenaza (2014: 105-106).

Siguiendo las aportaciones de las diferentes corrientes feministas, con el feminismo negro marxista y chicano (Davis, 2004; Anzaldúa, 2007), junto con el desarrollo de los feminismos decoloniales (Mohanty, 2008; Spivak, 2010), se llega a la conclusión de que no hay un feminismo único y universal y, por ende, un único sujeto mujer universal y abstracto. Según la clase y la raza, varían las condiciones de vida, necesidades y opresión de género. Así, se pone de relieve como universal supone finalizar con una mujer occidental, blanca y de clase media como referente, apostando por una interseccionalidad de relaciones de poder que oprimen al sujeto mujer y sus cuerpos, sobre las que también reflexionan las feministas posmodernas (De Lauretis, 2000; Braidotti, 2005; Butler, 2001) y que generan debates entre la necesidad de reconocer estas diferencias o primar la universalidad para garantizar una redistribución de derechos (Fraser, 2016).

Las economistas feministas constatan que todos los esfuerzos realizados desde las distintas perspectivas económicas -neoclásica, marxista o estudios de la cuantificación-, tienden a valorar el trabajo doméstico, «no consideran los nuevos aspectos de esta actividad y mantienen como referencia el trabajo asalariado» (Carrasco, 2017: 31). Aquí se da una dicotomía, ya que si no se asigna valor de mercado a las actividades domésticas se corre el riego de continuar invisibilizando a las mujeres pero, el problema es «cómo reconocer dicho trabajo sin otorgar un valor de cambio a todas las actividades no monetizadas, porque muchas no son comparables a la producción mercantil»(Carrasco, 2017: 34). Ante esto, Picchio justifica que «el trabajo doméstico permanece oculto porque sostiene los demás tipos de trabajo, formal e informal, asalariado y no asalariado» (2017: 204).

\section{Evolución de la «economía feminista»: líneas de investigación y propuestas}

A partir de la década de los 80 se da un avance en la «economía feminista» porque 120 se generaliza el uso de la categoría de «género» y se intenta replantear categorías como pro- 
ducción o trabajo, para eliminar la subvalorización que se tiene del trabajo de las mujeres. Se considera que los procesos de producción de bienes y servicios orientados a la subsistencia y reproducción de las personas deben incluirse en la actividad económica. Junto con esto, el concepto de reproducción pasa a ocupar un lugar central: se habla de «reproducción social» como un proceso dinámico que implica la reproducción biológica y de la fuerza de trabajo, la reproducción de bienes de cambio y de producción y la reproducción de las relaciones de producción (Carrasco, 2017:35).

Esto produce un cambio de perspectiva, la división sexual del trabajo pasa a analizarse teniendo en cuenta las interrelaciones que se generan dentro de un sistema en movimiento. De este modo, se hace frente a la separación artificial entre la esfera productiva y reproductiva de la economía clásica, se pone de manifiesto que el mercado laboral no es una entidad sexualmente neutra y que las relaciones de género están en la base de la organización del trabajo y la producción. Además, se desarrolla un marco de análisis que integra las esferas familiar, mercantil y pública, vistas como un todo social y desde el que realizar los estudios socioeconómicos de las mujeres. «No es posible captar la problemática en el mercado de trabajo si no se consideran las restricciones y condiciones familiares y la actuación de las políticas sociales» (Carrasco, 2006: 17).

Por otra parte, se observa cómo, pese a los intentos de incluir el género en las políticas macroeconómicas en un contexto de políticas de ajuste, continúan implementándose políticas bajo la protección del discurso económico dominante, que no tiene en cuenta las relaciones de poder y distintas experiencias entre mujeres y hombres'. Por ello, se inician progresivamente diversas líneas de investigación que van desde la feminización de la pobreza, la crítica a los modelos keynesianos por ser ciegos al sexo o al Estado de Bienestar por su sesgo de género, que hace que las mujeres tengan una ciudadanía social de menor categoría (Carrasco, 2017: 40-42).

También destaca otra línea de estudio que parte de la centralidad de la vida humana y la consecución de estándares de vida aceptables para la población que satisfagan necesidades biológicas, sociales, emocionales y afectivas, adaptándose a las identidades individuales y relaciones sociales. Finalmente, ha tenido un importante desarrollo todo lo relacionado con el trabajo de las mujeres. En cuanto al trabajo el hogar, se entiende que se trata de un trabajo diferente no comparable con las actividades realizadas en el mercado -cuyo objetivo era el cuidado de la vida y el bienestar de las personas del hogar y no el logro de beneficios-, donde las mujeres pasan de ser personas dependientes a activas y creadoras de valores y culturas distintos de los modelos masculinos.

1 Cabe destacar como en la década de los noventa se crea la International Association for Feminist Economics, que realizará una conferencia anual que ayuda a consolidar la «economía feminista». 
En este contexto, el análisis cada vez más profundo del contenido de las actividades desarrolladas en el hogar, llevó -de manera bastante natural-a plantear los aspectos subjetivos de las necesidades humanas y el importante papel que jugaba el "trabajo de cuidados". De esta manera, el "cuidado" [...] comenzó a emerger como un aspecto central del trabajo doméstico, como la dimensión fundamental que lo aleja del trabajo asalariado (Carrasco, 2006: 16).

Asimismo, con la incorporación progresiva de las mujeres al mundo laboral, se aprecia como son éstas las que asumen la doble jornada. Como consecuencia, se visibiliza el conflicto de conciliar tiempos y espacios y, por ende, la relación entre la producción capitalista, con su búsqueda de obtener beneficio, y la reproducción social de las personas. Se destapa cómo desde la teoría se había ocultado el mecanismo de reproducción de la población y de la fuerza de trabajo, relegando las actividades realizadas en el hogar al campo de lo «no económico». Con los estudios sobre el uso del tiempo se analiza la interrelación entre ambos trabajos y los efectos en las vidas de las mujeres (Carrasco y Mayordomo, 2017: 159).

Ante esto Carrasco y Mayordomo (2017: 159) subrayan que, para abordar esta situación no se debe plantear el trabajo familiar y el trabajo asalariado como un problema de estrategias femeninas de conciliación de tiempos, porque supone seguir reconociéndolo como una cuestión personal, privada y específica de las mujeres $y$, por otro lado, no comprender que es imposible que la gran parte de las mujeres se incorporen al mercado laboral de la forma en que lo hace la población masculina.

Por eso, abogan por aplicar una perspectiva global para abordar la complejidad de este fenómeno, de modo que considere la interrelación dinámica entre familia, mercado y Estado. "Los enfoques y análisis parciales centrados en el mercado laboral que relegan una actividad social fundamental -las tareas de relaciones y cuidados- a una categoría secundaria y sin importancia están incapacitados para ofrecer verdaderas alternativas de cambio» (Carrasco y Mayordomo, 2017: 159). Y es que desde la «economía feminista» se ha hecho hincapié en recuperar la dimensión relacional y afectiva del «estar-bien» (Pérez, 2014: 76), lo que se traduce en una complejización en las relaciones entre mercado-no mercado que exceden los análisis de coste-beneficio.

El trabajo de cuidados consiste en una actividad clave para el sostenimiento de la vida, «una experiencia femenina dedicada al cuidado de las personas a lo largo de todo su ciclo vital» (Carrasco y Díaz, 14: 2017), que pasó a ser el centro de la reproducción social y definió a las mujeres como sostenedoras de todo el entramado social y económico. Esto hizo visible la vulnerabilidad y necesidad de interdependencia entre las personas y la ecodependencia, tal y como también destacan las corrientes ecofeministas (Herrero, 2017; Mies y Shiva, 2016). 
Por tanto, para visibilizar el trabajo doméstico Picchio aboga por visibilizar todo el proceso de reproducción social dentro del marco de la estructura básica del sistema económico, mediante una estrategia «basada en una inversión de la relación producción-reproducción, inspirada por la idea de un desarrollo humano que convierta a la producción y los mercados en instituciones responsables del bienestar humano y obligadas a rendir cuentas» (2017: 202), donde se reconozca la dimensión material e inmaterial de las «desesidades» de vidas y su indisolubilidad (Pérez, 2014: 76).

Pero visibilizar el trabajo doméstico supone un «estorbo teórico» (Picchio, 2017: 203) a nivel estadístico, teórico y político es complejo, por lo que muchas veces se naturaliza o se confía a la familia. Por eso, paradójicamente, no es posible integrar el trabajo de reproducción de las mujeres en la teoría económica ocultando las diferencias entre el trabajo de producción de mercancía y el de reproducción de las personas, aunque es necesario reconocer esas diferencias en un contexto «macro». Valorar el trabajo en el hogar revela «la incapacidad conceptual y analítica de las estadísticas para manejar una definición aceptable de trabajo y el interés de una sociedad patriarcal de mantener oculta la actividad de las mujeres» (Carrasco, 2006: 18). Así lo revelan las economistas feminista al analizar herramientas como las estadísticas de empleo usada para la Encuesta de Población Activa o los indicadores del Producto Interior Bruto (PIB) (Carrasco, 2006). «De lo que se trata es de hacer uso de la experiencia de las mujeres en las redes sociales de reproducción e introducir cambios radicales en el sistema real y sus análisis» (Picchio, 2017: 203). Y es que el mercado puede generar

mucha insatisfacción vital, porque los mercados nos tratan como los seres autosuficientes que en el fondo no somos, niegan la dimensión relacional de la vida si no puedes pagarla y porque el mercado desregulado y precario no nos ofrece la identidad mercantilizada a la que aspiramos; pero no porque las empresas no hagan negocio con las desesidades ${ }^{2}$ afectivas, comunicativas y relacionales (Pérez, 2014: 76).

Por tanto, desde los cuidados se puede criticar la producción: con su visibilización se cuestiona todo el sistema económico desde la raíz de la vida cotidiana, son un enclave estratégico para descubrir «dónde y cómo se resuelve la vida en un sistema que la ataca y qué perversidades respecto a la propia vida encierra ese sistema, que se construye en torno al proceso de producción mercantil» (Pérez, 2014:207).

$2 \quad$ Pérez usa esta palabra, tomada a su vez en el contexto de la Educación Popular y la Investigación Acción Participativa en Centroamérica. Allí, las mujeres lanzan la propuesta de un nuevo vocablo "para resignificar la idea de "necesidades" sin escindirla de los "deseos": las "desesidades" [...] Se pasaban la vida luchando por los deseos de otros» (2014: 40), y ellas se olvidaban de los suyos propios. 
Por otra parte, estamos viendo como los procesos de globalización han creado una nueva división internacional del trabajo que está repercutiendo especialmente en el deterioro de las condiciones de vida y trabajo de las mujeres, una «feminización de la pobreza», una restructuración de la reproducción en beneficio de la acumulación del capital insostenible -especialmente por su impacto humano (Dalla Costa, 2009)-, un nuevo orden colonial. Al visibilizarse el trabajo doméstico no remunerado se ve mejor todo el proceso de reproducción social tanto en las economías de subsistencia tradicional del Sur como en las industriales del Norte. Es el caso de la «cadena global de cuidados» (Arruzza y Cirillo, 2017: 163), ejemplo claro de cómo el capitalismo, mediante su organización del trabajo y subordinación de la esfera reproductiva, "somete a mujeres que se encuentran en países distintos y en condiciones sociales diversas a una presión análoga, pero en medidas y grados diversos y con consecuencias significativamente diferentes» (Arruzza y Cirillo, 2017: 164).

Mies, siguiendo la línea de las teorías del patriarcado capitalista (Eisenstein, 1980; Von Wherlfhof, 2007) y del patriarcado del salario (Federici, 2017), resalta que «la construcción de la mujer como madre, mujer y ama de casa era el truco por el que el 50\% del trabajo humano estaba definido como recurso libre» (1998, Ix). Un trabajo invisibilizado y naturalizado que se trata como mercancía gratuita y en el que se aplica la violencia de forma más sofisticada, tanto en la esfera pública como privada, para estimular la acumulación primitiva del capital. Antes se realizaba mediante el pillaje de tierras y esclavos, hoy a través de las mujeres, excolonias y naturaleza -la base de la vida y la producción. Un proceso, pues, que afecta tanto a las personas asalariadas, como a las no asalariadas (mujeres, colonias, campesinado).

Para hacer frente a todas las consecuencias descritas anteriormente, desde la «economía feminista» se han lanzado diversas propuestas para hacer frente al sesgo androcéntrico de otros teorías económicas, como apostar por un modelo de «conflicto cooperativo», para analizar desde los modelos de negociación la situación de las personas tanto en la familia como en el trabajo asalariado. Otra iniciativa va más allá del análisis de la relación entre familia, mercado laboral y políticas públicas, ya que aboga por considerar el proceso de reproducción social el centro del análisis en vez del mercado (Carrasco, 2017: 46-47).

Por otro lado, para establecer medidas para reorganizar tiempos y espacios, se apuesta por elaborar y aplicar políticas públicas que incidan en el desarrollo de ayudas y/o servicios públicos de cuidados, presionando al sector masculino para que asuma la parte correspondiente de las tareas doméstico-familiares (Carrasco y Mayordomo, 2017: 161).

También se incide en la creación de presupuestos con enfoque de género, con el que se reconocerían las diferentes necesidades, privilegios, derechos, obligaciones y contribu- 
ciones de hombres y mujeres en la producción de bienes y su participación en los trabajos para la reproducción social. Es cierto que llevar a cabo esta herramienta es complicada tanto por el gasto para su realización, como por la poca aceptación por parte de las autoridades económicas de los gobiernos, pero puede suponer una herramienta muy poderosa tanto para la equidad socioeconómica, como para conseguir determinados estándares de vida, lo que conecta con situar los cuidados como centro del desarrollo humano (Carrasco, 2006: 20-22).

La importancia-además de simbólica- de estos enfoques es poner a la Administración Pública en relación directa con los estándares de vida de la población, destacando la importancia del trabajo de cuidados. De esta manera, se está asumiendo que existe una tensión histórica entre el bienestar de la población y el beneficio privado que exige la participación del sector público para la organización y redistribución de los recursos, en función de los estándares de vida generalizados (Carrasco, 2006: 22).

Así, con estos nuevos enfoques no sólo se da la misma importancia teórica a ambas esferas, sino que «plantean una ruptura con las aproximaciones tradicionales: abandonan el mercado como eje vertebrador de toda la actividad económica social y lo sustituyen por la esfera de la reproducción» (Borderías y Carrasco, 1994: 82).

Por tanto, se puede decir que la «economía feminista» cuestiona profundamente el discurso neoclásico; se recuperan los elementos del feminismo invisilizado como el trabajo de cuidados; se amplían las fronteras de la economía al incluir lo no mercantil, se critica la estructura dualista universalizante y jerárquica que confiere total reconocimiento al mundo público y la economía mercantil y se desarrollan nuevas estadísticas de acuerdo a los nuevos marcos analíticos (Carrasco, 2006: 23).

Ante esto, la apuesta de la «economía feminista» es clara «frente al conflicto capitalvida, apostar por la vida» (Carrasco y Díaz, 2017: 15). Pérez defiende subvertir la economía -heteropatriarcal, destructora a nivel medioambiental, colonialista y racista- donde todo gira alrededor del BBAh (hombre blanco, burgués, varón adulto y hererosexual) (Pérez, 2017), también llamado «homus economicus» (Carrasco, 2017) o Pequeño Hombre Blanco (Mies, 1886). Considera clave dinamitar el iceberg que, de modo metafórico, representa muy bien la forma en que se sustenta el capitalismo o "Cosa escandalosa» (Haraway, 1991: 340): en la parte visible están los mercados, regidos por la lógica de la acumulación del BBAh, mientras que en la parte oculta bajo el agua -la de mayores dimensiones se encuentran los procesos que giran en torno a vida. «Este iceberg pivota en torno a un eje heteropatriarcal y colonialista que define los niveles de visibilidad y los mecanismos de invisibilización» (Pérez, 2017: 31 ). 
En el momento en el que nos encontramos se aprecia la convivencia de propuestas basadas en lo que Pérez clasifica como «economías feminista de la conciliación» y «economía feminista de la ruptura» (2005, en Carrasco, 2006: 23), cuya diferencia es el grado de ruptura que establece respecto a los paradigmas androcéntricos dominantes. La primera -la de conciliación- reformula los marcos existentes pero se mantiene dentro, tratando de eliminar rasgos androcéntricos y compatibilizarse con nuevos conceptos y metodologías. En la segunda, se produce una ruptura total de las estructuras tratando de construir nuevos paradigmas que integran las actividades que participan en la reproducción social y el sostenimiento de la vida humana -entendida como una relación armónica no solo entre todos los seres humanos sino de estos con la naturaleza.

Pérez subraya que, en el actual contexto, se necesita iniciativas de transición, que se conjuguen con respuestas urgentes, para sostener las bases de un cambio sistémico. Para ello, no sólo deben reivindicarse luchas propiamente feministas -que deben dirigirse tanto a mujeres como a hombres-, sino incluir otras luchas que ya existen y que nos reconocen explícitamente como feministas, como la soberanía alimentaria o deuda. También se apuestan por la realización de experiencias concretas y de aplicación al campo de lo práctico; el establecimiento de diálogos entre iguales con mujeres que están realizando prácticas de «economía feminista» en diferentes partes del mundo sin distinción de clase, etnia o lugar. Por otro lado, también aboga por la creación de redes con otras economías críticas alternativas como la social y solidaria o la ecologista, entre otras (Carrasco y Díaz, 2017) (Pérez, 2017) y por la «cuidadanía», como una nueva forma de reconocerse los sujetos y de poner los cuidados en el centro frente a la ciudadanía, que ha sido la manera tradicional de reconocer a los sujetos en sociedades que ponen a los mercados capitalistas en el epicentro (Pérez, 2017: 221).

\section{Conclusiones}

Así, se ha comprobado a través de la labor de las diversas corrientes feministas en su aportación a la «economía feminista» como el capitalismo, junto con el patriarcado, homogeniza condiciones de vida, creando una respuesta de efecto universalizador ante el capital, al exponer a las mujeres las exigencias y consecuencias de la acumulación capitalista. Esta oposición al capitalismo como universalidad real puede ser la base para crear un «universalismo insurgente», basado en «una plena solidaridad política y de las luchas sociales, a la luz de un mismo objetivo compartido, dictado por la misma necesidad y deseo: superar el capitalismo rumbo a una sociedad más justa y a fin de hacer posible la liberación de las mujeres» (Arruzza y Cirillo, 2017: 164). 
Además, en relación a todos los avances, reflexiones y propuestas llevados a cabo por la «economía feminista» Orozco realiza una reflexión clave:

Si la lucha feminista durante décadas ha priorizado la cuestión de los trabajos, quizá ahora sea el momento de dar un giro: en lugar de entrar por la vía del trabajo, cuestionemos la economía por la vía de a qué necesidades/deseos responden esos trabajos. No se trata de cómo nos repartimos las tareas a hacer, sino de abrir la pregunta de qué es lo que necesitamos - queremos hacer. [...] ¿ Cuáles son las vidas que queremos sostener, cuál es la vida que merece ser vivida? Quizá sea este el núcleo duro de la subversión (2017: 57)

Lo que está claro es que las mujeres tienen un papel protagonista en un planteamiento basado en el desarrollo humano, tanto por su función en la reproducción, como por su experiencia política en hacer frente al conflicto inherente a la relación perversa entre la producción orientada hacia la obtención de un beneficio y la reproducción social. «Las mujeres están modificando el equilibrio de poder a través de la visibilidad de su trabajo no remunerado, la autoridad de su responsabilidad y las políticas de las necesidades y el deseo» (Picchio, 239: 2017), algo que se pudo contemplar claramente el 8 de marzo de 2019, donde miles de mujeres clamaban por subvertir este mundo patriarcal y neoliberal globalizado en el que vivimos, algo que sólo será posible desde los Feminismos.

\section{Bibliografía}

Amorós, Celia (2005). «La dialéctica del sexo de Shulamith Firestone: Modulaciones feministas del freudomarxismo» en AMOrós, C. y Ana De Miguel (eds.) (2005). Teoría feminista: de la llustración a la globalización. Del feminismo liberal a la posmodernidad. Vol. 2, Madrid: Minerva Ediciones, pp. 71-105.

ANZALDÚA, Gloria (2007). Bordelands/La Frontera, SN Francisco: Aunt Lute Books.

ArruzZA, Cinzia (2015). Las sin parte. Matrimonios y divorcios entre feminismo y marxismo, Barcelona: Editorial Sylone.

ArruzZa, Cinzia y Lidia CiRILL (2017). Dos siglos de feminismos. Los ejemplos más significativos, los problemas más actuales, Barcelona: Editorial Sylone.

Braidott, Rosi (2005). Metamorfosis. Hacia una teoría materialista del devenir, Madrid: Akal. Borderías, Cristina y Cristina CarRasco (1994). «Las mujeres y el trabajo: aproximaciones históricas, sociológicas y económicas» en Borderías, Cristina; Carrasco, Cristina y Carme Alemany (eds.) (1994). Las mujeres y el trabajo: rupturas conceptuales, Barcelona: Icaria, pp. 15-109. 
Butler, Judith (2001). El género en disputa, México: Paidós.

Carrasco, Cristina (2006). "La economía feminista: una apuesta por otra economía», pp 1-32. Disponible en: http://obela.org/system/files/CarrascoC.pdf (Fecha de consulta: 1/3/2019).

Carrasco, Cristina (2017). «Introducción: Hacia una economía feminista» en CARRASCO, Cristina (ed.) (2017). Mujeres y economía, Barcelona: Icaria Editorial, pp. 1 1-55.

Carrasco, Cristina y Carme díAZ (2017). «Presentación» en Carrasco, Cristina y Carme DIAZ (eds.) (2017). Economía feminista. Desafíos, propuestas, alianzas, Barcelona: Entrepueblos, pp. 13-20.

CarRasco, Cristina y Maribel maYORDOMO(2017). «Tiempos, trabajos y organización social: reflexiones en torno al mercado laboral femenino» en CARRASCO, Cristina (ed.) (2017). Mujeres y economía, Barcelona: Icaria Editorial, pp. 125-171.

Dalla Costa, Mariarosa (2009). Dinero, perlas y reproducción feminista. Madrid: Akal

Dalla Costa, Mariarosa y Selma James (1972). «The power of women and the subversion of the community» en https://libcom.org/. Disponible en: https://libcom.org/ files/Dalla\%20Costa \%20and\%20James\%20-\%20Women\%20and\%20the\%20 Subversion\%20of\%20the\%20Community.pdf (Fecha de consulta: 27/2/19).

De LaURetIS, Teresa (2000). Diferencias. Etapas de un camino a través del feminismo, Madrid: Horas y horas.

Delphy, Cristhine (1982). Por un feminismo materialista: el enemigo principal y otros textos, Barcelona: La Sal.

EISENTEIN, Zillah (1980). Patriarcado capitalista y feminismo socialista, México: Siglo XXI.

FeDERICl, Silvia (2017). El patriarcado del salario. Críticas feministas al Marxismo, Madrid: Traficantes de sueños.

FIRESTONE, Shulamith (1976). La dialéctica del sexo: en defensa de la revolución feminista, Barcelona: Kairós.

Fraser, Nancy y Judith Butler (2016). ¿2Redistribución o reconocimiento? Un debate entre marxismo y feminismo, Madrid: Traficantes de Sueños.

Gardiner, Jean (2017). «Los padres fundadores» en CARRASCO, Cristina (ed.) (2017). Mujeres y economía, Barcelona: Icaria Editorial, pp. 55-90.

HaraWAY, Donna J., Ciencia, cyborgs y mujeres: la reinvención de la naturaleza, Valencia, Universitat de Valencia, 1995.

HARTMANN, Heidi. «Un matrimonio mal avenido: hacia una unión más progresiva entre marxismo y feminismo» en Papers de la Fundació/88, pp. 1-32. Disponible en: http://www. fcampalans.cat/archivos/papers/88.pdf (Fecha de consulta: 28/2/19). 
Herrero, Yayo (2017). «Economía ecológica y economía feminista: un diálogo necesario» en Carrasco, Cristina y Carme Diaz (eds.) (2017). Economía feminista. Desafíos, propuestas, alianzas, Barcelona: Entrepueblos, pp. 121-142.

MACKINNON, Catharine (1995). Hacia una teoría feminista del Estado, Madrid: Cátedra.

MIES, Maria (1998). Patriarchy and accumulation on a world scale: women in the international division of labour, Londres: Zed Books.

MIES, Maria y Vandana SHIVA (2016). Ecofeminismo: teoría, crítica y perspectivas, Barcelona: Icaria.

Milletr, Kate (1995). Política sexual, Madrid: Cátedra: Instituto de la Mujer.

Mitchell, Juliet (1977). La condición de la mujer, Barcelona: Anagrama.

MOHANTY, Chandra Talpade (2008). «De vuelta a Bajo los ojos de Occidente: La solidaridad feminista a través de las luchas anticapitalistas» en SUÁrEZ-NAVAS, Liliana y Regina Berger (eds.) (2008). Descolonizando el feminismo: teorías y prácticas desde los márgenes, Madrid: Cátedra, pp. 404-454.

MouINA, Cristina (2005). «El feminismo socialista estadounidense desde la "Nueva lzquierda". Las teorías del Sistema Dual (Capitalismo+Patriarcado)» en AMORÓs, C. y Ana De MigueL (eds.) (2005). Teoría feminista: de la llustración a la globalización. Del feminismo liberal a la posmodernidad. Vol. 2, Madrid: Minerva Ediciones, pp. 149-187.

MORGAN, Robin (1985). Sisterhood is global: the international women's movement anthology, Harmondsworth: Penguin Books.

Ouva, Asunción (2005). "La teoría de las mujeres como clase social: Christine Delphy y Lidia Falcón » en Amorós, C. y Ana De Miguel (eds.) (2005). Teoría feminista: de la llustración a la globalización. Del feminismo liberal a la posmodernidad. Vol. 2, Madrid: Minerva Ediciones, pp. 109-146.

PéreZ, Amaia (2017). «¿Espacios económicos para la subversión feminista?» en CarRasco, Cristina y Carme DIAZ (eds.) (2017). Economía feminista. Desafíos, propuestas, alianzas, Barcelona: Entrepueblos, pp. 29-58.

PICCHIO Antonella (2017). «Visibilidad analítica y política del trabajo de reproducción social» en Carrasco, Cristina (ed.) (2017). Mujeres y economía, Barcelona: Icaria Editorial, pp. 201-242.

Puleo, Alicia (2005). «Lo personal es político: el surgimiento el feminismo radical» en AMORós, C. y Ana De Miguel (eds.) (2005). Teoría feminista: de la llustración a la globalización. Del feminismo liberal a la posmodernidad. Vol. 2, Madrid: Minerva Ediciones, pp. 37-67.

SPIVAK, Gayatri Chakravorty (2010). Crítica de la razón poscolonial: hacia una historia del presente evanescente, Madrid: Akal. 
Young, Iris (1992). "Marxismo y feminismo, más allá del "matrimonio infeliz" (una crítica al sistema dual)» en El cielo por asalto, N4, pp. 43-69. Disponible en: http:// www.democraciasocialista.org/wp-content/uploads/2014/03/139104361-YoungMarxismo-y-feminismo.pdf (Fecha de consulta: 28/2/19).

VON WerlfHOF, Claudia (2007). «No Critique of Capitalism without a Critique of Patriarchy» en CNS - Capitalism - Nature - Socialism, Vol. 18, Nr. 1, New York/London (Routledge), March 2007, pp. 13-27. Disponible en: http://emanzipationhumanum. de/downloads/critique.pdf (Fecha de consulta: 27/2/2019). 